\title{
Echocardiography and pulmonary embolism severity index have independent prognostic roles in pulmonary embolism
}

\author{
Olivier Sanchez ${ }^{1}$, Ludovic Trinquart ${ }^{2}$, Benjamin Planquette ${ }^{1}$, Francis Couturaud $^{3}$, \\ Franck Verschuren ${ }^{4}$, Vincent Caille ${ }^{5}$, Nicolas Meneveau ${ }^{6}$, Gérard Pacouret ${ }^{7}$, \\ Pierre-Marie Roy ${ }^{8}$, Marc Righini ${ }^{9}$, Arnaud Perrier ${ }^{9}$, Laurent Bertoletti ${ }^{10}$, \\ Florence Parent ${ }^{11}$, Christine Lorut ${ }^{12}$ and Guy Meyer ${ }^{1}$
}

Affiliations: 'Université Paris Descartes, Sorbonne Paris Cité, Assistance Publique Hôpitaux de Paris, Hôpital Européen Georges Pompidou, Service de Pneumologie et Soins Intensifs, INSERM U 765, Paris, ${ }^{2}$ Université Paris Descartes, Assistance Publique Hôpitaux de Paris, Hotel Dieu de Paris, INSERM CIE 4, Paris, ${ }^{3}$ Université Européenne de Bretagne, Université de Brest, EA3878, IFR148, Hôpital La Cavale Blanche, Service de Pneumologie, Brest, ${ }^{5}$ Hôpital Ambroise Paré, Service de Réanimation médicale, Assistance Publique Hôpitaux de Paris, Boulogne, 'Université de Franche Comté, EA 3920, IFR 133, CHU Jean Minjoz, Service de Cardiologie, Besançon, ${ }^{7} \mathrm{Hôpital}$ Trousseau, Service de Cardiologie A, CHRU de Tours, Chambray-lès-Tours, ${ }^{8} \mathrm{CHU}$ d'Angers, Service d'accueil des urgences, Angers, ${ }^{10} \mathrm{CHU}$ St-Etienne, Service de Médecine Interne et Thérapeutique, Groupe de Recherche sur la Thrombose (EA3065), St-Etienne, ${ }^{11} \mathrm{Hôpital}$ Bicêtre, service de pneumologie, Assistance Publique Hôpitaux de Paris, Le Kremlin Bicêtre, and ${ }^{12}$ Hôtel Dieu de Paris, service de pneumologie, Assistance Publique Hôpitaux de Paris, Paris, France. "Université Catholique de Louvain, Cliniques Universitaires Saint-Luc, Acute Medicine Dept, Accidents and Emergency Unit, Brussels, Belgium. ${ }^{9}$ Geneva University Hospital, Division of General Internal Medicine, Geneva, Switzerland.

Correspondence: 0. Sanchez, Service de Pneumologie et Soins Intensifs, Hôpital Européen Georges Pompidou, 20 rue Leblanc 75015 Paris, France. E-mail: olivier.sanchezdegp.aphp.fr

ABSTRACT We analysed a cohort of patients with normotensive pulmonary embolism (PE) in order to assess whether combining echocardiography and biomarkers with the pulmonary embolism severity index (PESI) improves the risk stratification in comparison to the PESI alone.

The PESI was calculated in normotensive patients with PE who also underwent echocardiography and assays of cardiac troponin I and brain natriuretic peptide. 30-day adverse outcome was defined as death, recurrent PE or shock.

529 patients were included, 25 (4.7\%, 95\% CI 3.2-6.9\%) had at least one outcome event. The proportion of patients with adverse events increased from $2.1 \%$ in PESI class I-II to $8.4 \%$ in PESI class III-IV, and to $14.3 \%$ in PESI class V $(\mathrm{p}<0.001)$. In PESI class I-II, the rate of outcome events was significantly higher in patients with abnormal values of biomarkers or right ventricular dilatation. In multivariate analysis, the PESI (class III-IV versus I-II, OR 3.1, 95\% CI 1.2-8.3; class V versus I-II, OR 5.5, 95\% CI 1.5-25.5 and echocardiography (right ventricular/left ventricular ratio, OR (for an increase of 0.1 ) 1.3, 95\% CI 1.1-1.5) were independent predictors of an adverse outcome.

In patients with normotensive PE, biomarkers and echocardiography provided additional prognostic information to the PESI.

$\mathbf{0}$

@ERSpublications

In patients with normotensive $\mathrm{PE}$, biomarkers and echocardiography provided additional prognostic information to the PESI http://ow.ly/lJ8pW

Received: June 202012 | Accepted after revision: Oct 312012 | First published online: Dec 202012

Support statement: The study was funded by a grant of the Chancellerie des Universites (Legs Poix).

Conflict of interest: None declared.

Copyright @ERS 2013 


\section{Introduction}

Early mortality from pulmonary embolism (PE) depends on the clinical consequences of PE and on the underlying disease [1]. Risk stratification of patients with PE may enable definition of a low-risk group that may be treated on an outpatient basis and a high-risk group that should be admitted to hospital for close medical supervision [2]. The pulmonary embolism severity index (PESI), a clinical rule based on 11 clinical variables, defines five classes of patients with PE with different mortality rates at 90 days [3]. The PESI has been validated in several large cohorts and is now available as a simplified version based on seven variables [4-6]. Right ventricular dysfunction, assessed by either echocardiography or spiral computed tomography, and high levels of biomarkers, including cardiac troponin I (cTnI) and brain natriuretic peptide (BNP), have been linked to an increased risk of death or adverse outcome in patients with PE [7-9]. Little is known about the respective roles of the clinical findings summarised in the PESI, echocardiography and biomarkers for the risk assessment of patients with PE. We analysed the results of a large prospective multicentre cohort of consecutive patients with $\mathrm{PE}$ in order to determine whether the combination of echocardiography and biomarkers with the PESI improves the risk stratification of patients with PE compared with the PESI alone [8]. Cardiogenic shock at admission represents one of the most important prognostic factors in patients with PE and, according to the current guidelines of the European Society of Cardiology, it is widely admitted that further risk stratification by biomarkers, echocardiography or the PESI is not needed in these patients [2]. Therefore, we focused our study on patients with normotensive PE.

\section{Material and methods}

\section{Patients}

Consecutive adult patients with symptomatic normotensive PE admitted in the 11 participating sites in France, Belgium and Switzerland were recruited for the study. Patients were eligible if their PE was objectively confirmed according to current guidelines, as previously reported $[2,8]$. Patients were ineligible for the study if they had received therapeutic doses of anticoagulant treatment for $>24 \mathrm{~h}$ or had cardiogenic shock at admission defined by at least one of the following criteria: systolic blood pressure $<90 \mathrm{mmHg}$, signs of end-organ hypoperfusion or a need for catecholamine administration to maintain systolic blood pressure $>90 \mathrm{mmHg}$. Because the role of thrombolytic therapy remains unclear in clinically stable patients, we excluded patients with normotensive PE who received fibrinolytic treatment. Demographic data, symptoms and risk factors for venous thromboembolism, including all variables of the PESI, were obtained at the time of admission. Patients were managed according to the usual practices of each participating site by physicians blinded to the results of cardiac biomarkers.

The study protocol strongly recommended transthoracic echocardiography within $24 \mathrm{~h}$ of PE diagnosis by an experienced physician unaware of the results of cardiac biomarker determinations. The end-diastolic diameters of the right and left ventricles were measured along the long axis of the parasternal view and the right ventricle/left ventricle ratio was calculated [8].

On admission, blood samples were collected in heparinised tubes for the cTnI assay and in EDTA tubes for $\mathrm{BNP}$ determination, tubes were centrifuged and the resulting plasma was frozen and stored at $-80^{\circ} \mathrm{C}$. At the end of the study, circulating levels of $\mathrm{CTnI}$ and BNP were determined in a central laboratory by investigators blind to the patients' baseline characteristics and clinical outcome. cTnI levels were determined with quantitative photometric immunoassays using a Dimension-RxL Max analyser (Dade-Behring, Siemens Healthcare Diagnostics, Erlangen, Germany), according to the manufacturer's instructions. The lower limit of detection of the cTnI assay was $0.04 \mu \mathrm{g} \cdot \mathrm{L}^{-1}$. BNP levels were determined with an electrochemiluminescence immunoassay (BNP-Triage Biosite assay; Biosite Inc., San Diego, CA, USA) on a DxI analyser (Beckman Coulter Inc., Brea, CA, USA). The lower limit of detection in the BNP assay was $10 \mathrm{ng} \cdot \mathrm{L}^{-1}$.

\section{Outcomes}

30-day clinical follow-up data were obtained for all patients. Adverse clinical events were defined as allcause death, secondary cardiogenic shock as previously defined, or objectively confirmed symptomatic recurrent venous thromboembolism. All adverse events and the cause of death (i.e. related or unrelated to $\mathrm{PE}$ ) were adjudicated by an independent committee of two physicians unaware of the results of the initial clinical examination, echocardiography and biomarker determinations.

The study was an academic trial sponsored by the Délégation à la Recherche Clinique d'Ile de France. The funding body had no role in the design of the study, data analysis, or drafting the manuscript. The study was approved by local ethics committees (Ile de France II, Saint-Luc University Hospital and Geneva University Hospital committees for the French, Belgian and Swiss centres, respectively). All patients signed a written informed consent form. 


\section{Statistical methods}

Categorical variables were summarised using numbers and percentages, and continuous variables using median (interquartile range). PESI score was categorised into low risk (PESI class I or II), intermediate risk (PESI class III or IV) and high risk (PESI class V) categories. Proportions of deaths and adverse events according to the PESI risk class were compared using Chi-squared tests; median values of markers according to PESI risk class were compared using Kruskall-Wallis tests.

Univariate analyses, based on Chi-squared tests or t-tests, were performed. Independent associations with the outcome were assessed by including variables with a significance level of $\mathrm{p}<0.20$ on univariate analysis in a multivariate logistic regression model. Variables associated with the outcome at a significance level of $\mathrm{p}<0.05$ in backward stepwise regression analysis were retained. For the multivariate analysis, we conducted multiple imputation analysis to ensure that the results were robust for missing data. For each variable, we further estimated the proportion of explained variation (PEV) and partial PEV. The PEV measures the proportion of variation of the outcome variable that can be attributed to the variable, relative to the total variation of the outcome variable. Partial PEV measures the decline in explained variation when removing the prognostic factor from the model containing the other four factors.

In order to determine if echocardiography and biomarkers improved the risk stratification, we first constructed box plots showing the distributions of biomarkers and echocardiography values within each PESI risk class and among patients who did, or did not, experience an adverse event. In subsequent analyses, echocardiography and biomarkers were dichotomised according to thresholds validated in previous studies. We defined a BNP concentration $>100 \mathrm{ng} \cdot \mathrm{L}^{-1}$ as positive $\mathrm{BNP}[10,11]$, a cTnI concentration $>0.1 \mu \mathrm{g} \cdot \mathrm{L}^{-1}$ as positive $\mathrm{cTnI}[10,12]$ and a right ventricle/left ventricle ratio $>0.9$ as a positive right ventricle/left ventricle ratio $[10,12]$. We then cross-classified patients according to their PESI risk category and then according to their right ventricle/left ventricle ratio or biomarker risk category, each in turn. Within each sub-stratum, we estimated the risk of an adverse event. Finally, logistic regression analyses were conducted to determine possible links between prognostic factors (PESI risk category, BNP, cTnI and right ventricle/ left ventricle end-diastolic diameter ratio) and the risk of an adverse event.

\section{Results}

Patients

A total of 592 consecutive patients were included in the study between January 2006 and May 2007. 41 patients were excluded because of cardiogenic shock at inclusion. 22 haemodynamically stable patients were excluded because they were receiving fibrinolytic treatment. None of these normotensive patients experienced a 30-day adverse event. The final study population, therefore, comprised 529 patients with normotensive PE (table 1). At inclusion, 528 patients (99\%) were receiving an anticoagulant treatment. Fibrinolytic therapy was administered to eight patients (1.5\%) because of secondary cardiogenic shock. A vena cava filter was inserted in 22 patients (4\%).

\section{Outcome}

The 30-day prospective follow-up was complete for all patients. During follow-up, 25 patients (4.7\%, 95\% CI 3.2-6.9\%) suffered adverse events: death in 15 cases $(2.8 \%$, 95\% CI $1.7-4.6 \%)$, secondary cardiogenic shock in $15(2.8 \%)$ and recurrent venous thromboembolism in eight patients (1.5\%). One patient may have had several events qualifying for complicated outcome.

\section{PESI}

The PESI was calculated for all 529 patients; among them, 329 (62\%) were at low risk (PESI class I or II), 179 patients (34\%) were at intermediate risk (PESI class III or IV) and 21 patients (4\%) were at high risk (PESI class V). The mortality rate and the rate of adverse outcomes, including mortality, secondary cardiogenic shock and recurrent PE, increased significantly from $0.6 \%$ and $2.1 \%$ in the low-risk category to $9.5 \%$ and $14.3 \%$ in the high-risk category, respectively (table 2).

\section{Biomarkers and echocardiography}

Plasma concentrations of BNP and cTnI, and the right ventricle/left ventricle ratio increased significantly with the PESI (table 2).

\section{Predictors of adverse outcome}

Figure 1 shows the number and proportion of patients with an adverse outcome at 30 days in the six categories defined by cross-tabulation of the PESI risk class (low, intermediate or high risk) and the right ventricular dysfunction marker category (normal or elevated biomarkers; normal or abnormal right ventricle/left ventricle ratio). All biomarkers and echocardiography results enabled patients belonging to the 
TABLE 1 Characteristics of the 529 patients with pulmonary embolism

\begin{tabular}{|c|c|c|c|c|}
\hline \multirow[t]{2}{*}{ Characteristics } & \multirow[t]{2}{*}{ Available data } & \multirow[t]{2}{*}{ All } & \multicolumn{2}{|c|}{ 30-day adverse event } \\
\hline & & & Yes & No \\
\hline Patients & & 529 & 25 & 504 \\
\hline Age years & 529 & $67(52-77)$ & $70(60-81)$ & $67(51-77)$ \\
\hline Male & 529 & 247 (47) & $12(48)$ & $235(47)$ \\
\hline Cancer & 529 & $77(15)$ & $7(28)$ & $70(14)$ \\
\hline Altered mental state & 529 & $7(1)$ & $1(4)$ & $6(1)$ \\
\hline Syncope & 529 & $24(5)$ & $2(8)$ & $22(4)$ \\
\hline Heart rate beats $\cdot \min ^{-1}$ & 526 & $88(75-100)$ & 90 (80-103) & $88(74-100)$ \\
\hline Systolic blood pressure $\mathrm{mmHg}$ & 529 & $134(120-150)$ & $130(124-140)$ & $135(120-150)$ \\
\hline Cardiogenic shock on admission & 529 & $0(0)$ & $0(0)$ & $0(0)$ \\
\hline PESI class & 529 & & & \\
\hline $\mid$ ( $\leqslant 65$ points $)$ & & $131(25)$ & $1(4)$ & $130(26)$ \\
\hline II (66-85 points) & & 198 (37) & $6(24)$ & 192 (83) \\
\hline III (86-105 points) & & $109(21)$ & $10(40)$ & 99 (20) \\
\hline IV (106-125 points) & & 70 (13) & $5(20)$ & $65(13)$ \\
\hline$V(>125$ points $)$ & & $21(4)$ & $3(12)$ & $18(4)$ \\
\hline \multicolumn{5}{|l|}{ Echocardiography and cardiac biomarkers } \\
\hline$B N P n g \cdot L^{-1}$ & 521 & $71.0(27.0-210.0)$ & $275.0(69.0-431.0)$ & $67.0(27.0-190.0)$ \\
\hline Cardiac troponin $\mathrm{I} \mathrm{mg} \cdot \mathrm{L}^{-1}$ & 517 & $0.01(0.00-0.06)$ & $0.11(0.01-0.34)$ & $0.01(0.00-0.05)$ \\
\hline Right ventricle/left ventricle EDD ratio & 484 & $0.65(0.53-0.79)$ & $0.76(0.63-1.11)$ & $0.65(0.53-0.79)$ \\
\hline
\end{tabular}

Data are presented as $\mathrm{n}$, median (interquartile range) or $\mathrm{n}$ (\%). PESI: pulmonary embolism severity index; BNP: brain natriuretic peptide; EDD: enddiastolic diameter.

low-risk category (PESI class I or II), to be further stratified into two subgroups, one with a very low risk $(\leqslant 1 \%)$ of an adverse outcome and one with a $6 \%$ risk of 30 -day adverse outcome, with significant differences in the event rate between the subgroups with normal and abnormal biomarker and right ventricle/left ventricle ratio values ( $\mathrm{p}=0.03$ for $\mathrm{BNP} ; \mathrm{p}<0.001$ for $\mathrm{cTnI}$ and $\mathrm{p}<0.001$ for right ventricle/left ventricle ratio) (fig. 1 ). In the intermediate risk group of patients (PESI class III-IV), elevated biomarkers and right ventricular dilatation on echocardiography were associated with higher rates of adverse outcomes, but the difference to patients having normal values was significant for $\mathrm{cTnI}$ only $(\mathrm{p}=0.02$ for $\mathrm{cTnI} ; \mathrm{p}=0.42$ for BNP and $\mathrm{p}=0.9$ for echocardiography) (fig. 1). In the high-risk group (PESI class V), differences in the event rates between patients with normal and abnormal biomarker values and echocardiography were less pronounced and not significant, but the patient population was small (fig. 1).

TABLE 2 Rate of adverse outcomes and value of biomarkers and echocardiography according to the pulmonary embolism severity index (PESI) classes

PESI class

p-value

\begin{tabular}{cccc}
\hline I-II (low risk) & $\begin{array}{c}\text { III-IV (intermediate } \\
\text { risk) }\end{array}$ & V (high risk) & \\
329 & 179 & 21 & \\
$2(0.6)$ & $11(6.1)$ & $2(9.5)$ & $<0.001$ \\
$7(2.1)$ & $15(8.4)$ & $3(14.3)$ & $<0.001$ \\
& & & $<0.001$ \\
$44.5(21.5-146.0)$ & $126.5(46.0-299.0)$ & $221.0(130.0-385.0)$ & $<0.01$ \\
$0.01(0.00-0.04)$ & $0.01(0.00-0.10)$ & $0.03(0.00-0.19)$ & 0.02 \\
$0.63(0.51-0.76)$ & $0.71(0.56-0.86)$ & $0.59(0.51-0.88)$ &
\end{tabular}

Patients
End-points
30-day mortality
30-day adverse event
Echocardiography and cardiac biomarkers
BNP $\mathrm{ng} \cdot \mathrm{L}^{-1}$
Cardiac troponin I $\mu \mathrm{g} \cdot \mathrm{L}^{-1}$
Right ventricle/left ventricle EDD ratio

Data are presented as n, $\mathrm{n}(\%)$ or median (interquartile range), unless otherwise stated. Distributions of deaths and adverse events according to PESI risk class were compared using Chi-squared tests; distributions of markers according to PESI risk class were compared using KruskallWallis tests. BNP: brain natriuretic peptide; EDD: end-diastolic diameter. 

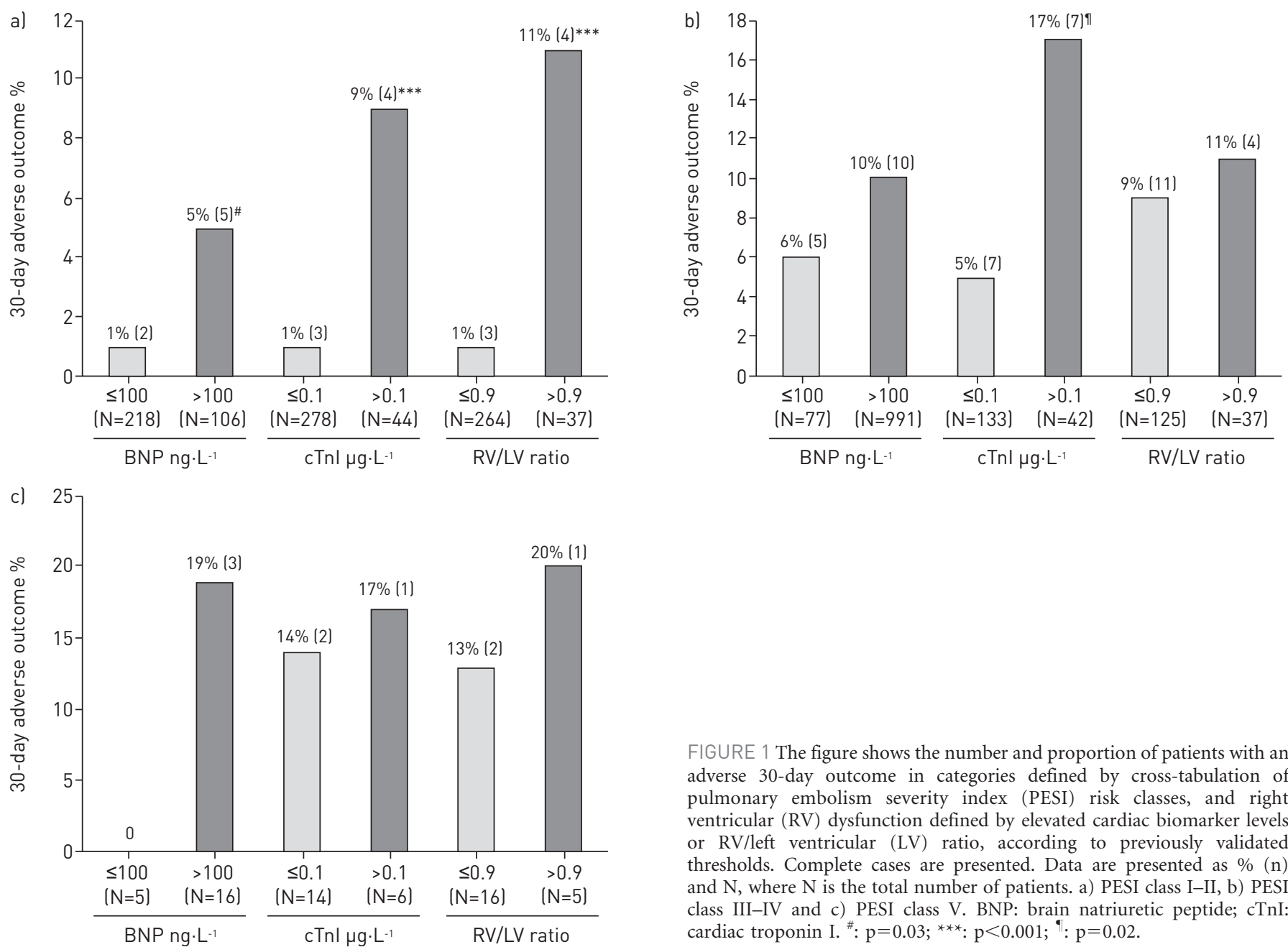

FIGURE 1 The figure shows the number and proportion of patients with an adverse 30-day outcome in categories defined by cross-tabulation of pulmonary embolism severity index (PESI) risk classes, and right ventricular (RV) dysfunction defined by elevated cardiac biomarker levels or RV/left ventricular (LV) ratio, according to previously validated thresholds. Complete cases are presented. Data are presented as \% (n) and $\mathrm{N}$, where $\mathrm{N}$ is the total number of patients. a) PESI class I-II, b) PESI class III-IV and c) PESI class V. BNP: brain natriuretic peptide; cTnI: cardiac troponin I. *: $\mathrm{p}=0.03 ;{ }^{* *}: \mathrm{p}<0.001 ;{ }^{\uparrow}: \mathrm{p}=0.02$.

Results of univariate logistic regression analyses showed that the PESI, BNP and right ventricle/left ventricle ratio were significantly associated with the occurrence of an adverse outcome (table 3). Multivariate analysis showed that, in addition to the PESI risk class, echocardiography but not biomarkers remained independent predictors of adverse outcome at day 30 in the study population (table 4). The variables included in the model accounted for about $10 \%$ of the variation in individual 30 -day outcomes; the proportions of variance explained by each right ventricular dysfunction marker (as well as partial PEV) were similar to those explained by the PESI risk class. Finally, multiple imputation analysis provided estimates consistent with the complete case analysis (table 4). The PESI was associated with a moderate prognostic sensitivity (72\%) and high negative predictive value (98\%) (table 5). Interestingly, the combination of PESI with right ventricle/

\begin{tabular}{|c|c|c|c|}
\hline Variable & Complete cases $\mathrm{n}$ & OR $(95 \% \mathrm{Cl})$ & p-value \\
\hline PESI class & 529 & & $<0.01$ \\
\hline III-IV versus I-II & & $4.2(1.7-10.5)$ & \\
\hline V versus I-II & & $7.7(1.8-32.1)$ & \\
\hline BNP\# & 521 & $1.3(1.1-1.6)$ & $<0.01$ \\
\hline Cardiac troponin I & 517 & $1.2(0.9-1.5)$ & 0.20 \\
\hline Right ventricle/left ventricle EDD ratio ${ }^{+}$ & 484 & $1.3(1.2-1.5)$ & $<0.0001$ \\
\hline
\end{tabular}

PESI: pulmonary embolism severity index; BNP: brain natriuretic peptide; EDD: end-diastolic diameter. ${ }^{\#}$ : for an increase of $250 \mathrm{ng} \cdot \mathrm{L}^{-1}$; ${ }^{9}$ : for an increase of $0.7 \mu \mathrm{g} \cdot \mathrm{L}^{-1} ;{ }^{+}$: for an increase of 0.1 . 
TABLE 4 Multivariate analysis

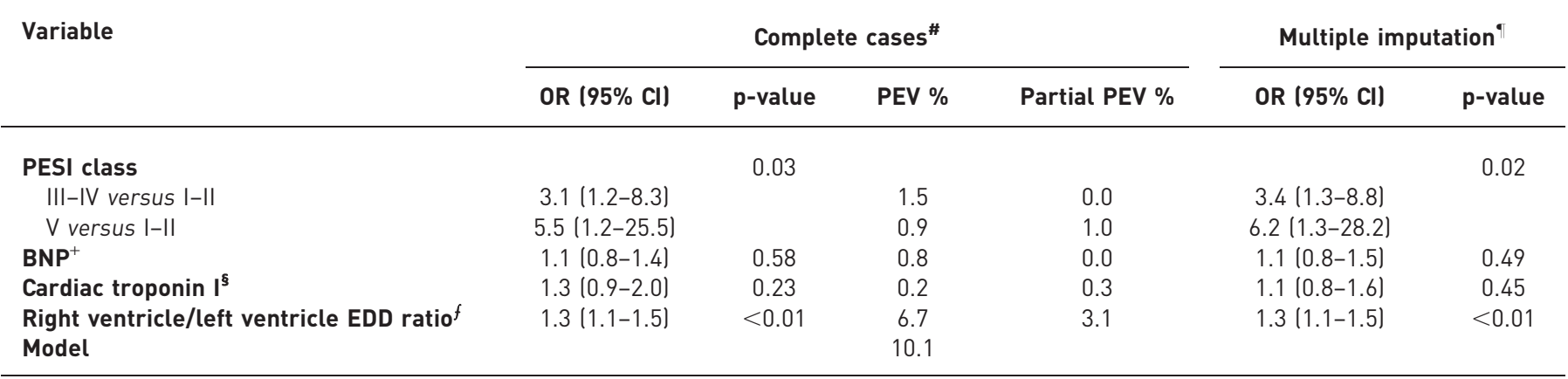

Missing values for any prognostic variable (heart rate $n=3$, respiratory rate $n=71$, temperature $n=4$, arterial oxyhaemoglobin saturation $n=4$ ) were assumed to be normal, a strategy used in the original determination of the pulmonary embolism severity index (PESI) [3]. PEV: proportion of explained variation, i.e. the amount of variation of the outcome variable that is attributable to the variable, relative to the total variation of the outcome variable; partial PEV measures the decline in explained variation when removing the prognostic factor from the model containing all four other factors; BNP: brain natriuretic peptide; EDD: end-diastolic diameter. ${ }^{\#}: \mathrm{n}=473 ;{ }^{\natural}: \mathrm{n}=529{ }^{+}{ }^{+}$: for an increase of $250 \mathrm{ng} \cdot \mathrm{L}^{-1}$; ${ }^{\S}$ : for an increase of $0.7 \mu \mathrm{g} \cdot \mathrm{L}^{-1} ;{ }^{f}$ : for an increase of 0.1 .

left ventricle ratio had a higher sensitivity and negative predictive value for predicting 30-day complicated outcome (table 5).

\section{Discussion}

This study shows that in patients with normotensive PE, right ventricular dysfunction on echocardiography provides prognostic information that is independent of clinical findings summarised by the PESI. In patients considered at low-risk according to the PESI, echocardiography and biomarkers identified two subgroups with different risks of adverse events. Among patients belonging to PESI class I or II, those with normal echocardiography results and normal levels of biomarkers had a very low risk of adverse events, which was significantly lower than the rate observed in patients with an abnormal echocardiography or elevated levels of biomarkers.

Five groups of patients with different risks of death were described in the original report describing the PESI. Subsequently, class I and II patients were combined in a low-risk group and were selected as possible candidates for outpatient treatment [13] and patients in class III to V were considered as "high-risk" patients $[5,6]$. In the present study, three groups were considered: one "low-risk" group corresponding to the usual low-risk group (defined as PESI class I or II), an "intermediate-risk" group defined as PESI class III or IV patients, and a "high-risk" group defined as PESI class V patients. This was done to comply with the recommendations of the European Society of Cardiology, in which three groups are considered [2]. Clustering the high-risk and intermediate groups or using the simplified PESI did not change the results (data not shown). Previous studies have compared the clinical approach based on the PESI and biomarkers or echocardiography for the risk stratification of PE with varying results. The respective values of $\mathrm{CTnI}$ and the original PESI for the prediction of mortality at 30 days were evaluated in a monocentric cohort of 567 patients with clinically stable PE [14]. In this study, the combination of cTnI with the clinical variables did not improve the negative predictive value of the PESI for the risk of 30-day mortality, but the other major adverse events, such as recurrent PE or cardiogenic shock, were not evaluated [14]. The prognostic value of the new high sensitivity troponin $\mathrm{T}$ (hsTnT) assay and of the simplified PESI was evaluated in a multicentre study including 526 normotensive patients with PE [15]. The hsTnT assay was associated with a high prognostic sensitivity and negative predictive value comparable to those of the simplified PESI. hsTnT and

TABLE 5 Value of pulmonary embolism severity index (PESI) alone and right ventricle/left ventricle (RV/LV) ratio alone and in combination for predicting a 30-day complicated outcome

\begin{tabular}{lcccc} 
& Sensitivity & Specificity & Positive predictive value & Negative predictive value \\
\hline PESI class III-IV-V & $72(18 / 25)$ & $64(294 / 459)$ & 10 & 98 \\
RV/LV $>\mathbf{0 . 9}$ & $36(9 / 25)$ & $85(389 / 459)$ & 11 & 96 \\
PESI class III-IV-V and RV/LV ratio $>\mathbf{0 . 9}$ & $88(22 / 25)$ & $57(261 / 459)$ & 10 & 99 \\
\hline
\end{tabular}

Data are presented as $\%(n / N)$ or $\%$. 
the simplified PESI were identified as independent predictors of 30-day complicated outcome defined by all-cause death or secondary shock or cardiopulmonary resuscitation [15]. Interestingly, the combination of elevated hsTnT with high risk simplified PESI had a sensitivity and negative predictive value of $100 \%$ [15]. By contrast, none of the patients with low-risk simplified PESI and low levels of hsTnT experienced an adverse outcome, but the authors did not report whether patients with low-risk simplified PESI and elevated hsTnT had a higher rate of adverse outcomes [15]. Recently, the simplified PESI has been compared to a risk-stratification method based on echocardiography and biomarkers proposed by recent European Society of Cardiology recommendations [12]. The combined end-point of all-cause mortality, objectively confirmed non-fatal symptomatic recurrent venous thromboembolism or non-fatal major bleeding was lower in the low-risk group identified by the simplified PESI than in the low-risk group identified by normal echocardiography findings and biomarker assays [12]. An approach combining clinical findings, echocardiography and biomarkers was not tested in this study. In another monocentric study, it was shown that right ventricular dysfunction and/or a high cTnI level increased the risk of death in patients classified as intermediate risk according to the PESI, whereas this was not observed in low-risk patients [16]. The additional role of echocardiography and biomarkers in predicting other major complications was not tested in this study.

Recently, AUJESKY et al. [13] demonstrated in an open-label multicentre randomised trial that outpatient treatment is not inferior to inpatient care in terms of efficacy and safety in selected low-risk patients (PESI class I or II). In this study, neither echocardiography nor biomarkers were used for the risk stratification, but more than two-thirds of the patients included were in the very low risk PESI class I [13]. Our results suggest that among patients belonging PESI class I or II, those with elevated levels of biomarkers or abnormal echocardiography results could require hospitalisation instead of outpatient care. However, our results must be confirmed in large independent cohort studies before being used to make a therapeutic decision.

The present study has several strengths; it was multicentre, enabling greater generalisation of the results, and patients were consecutively and prospectively recruited, limiting the possibility of major biases. All outcomes were assessed by an independent central committee whose members were unaware of the initial clinical data and results of echocardiography and biomarkers. The biomarkers were measured at the end of the study and the responsible physicians were also unaware of the results of biomarker assays and these results did not influence initial treatment. Conversely, participating physicians were aware of echocardiography results and their initial treatment option, i.e. anticoagulation alone or anticoagulation and thrombolysis may have been influenced by the results of echocardiography; this is why normotensive patients who received thrombolytic treatment were excluded from the analysis. The study was also limited by the low number of events and by the small number of patients in the intermediate and high-risk categories. This may explain why the difference between the proportion of intermediate-risk patients with outcome having abnormal echocardiography results or elevated biomarkers compared to those with normal results, was not statistically significant.

In conclusion, if confirmed in independent cohort studies, the present findings suggest that cardiac biomarkers and/or echocardiography will enable patients with a PESI I-II to be further stratified into two subgroups, one with a very low level of adverse outcome that can be safely treated as outpatients and one with a slightly higher risk requiring hospitalisation. The role of biomarkers and echocardiography in patients at intermediate or high risk according to the PESI is more questionable and should be evaluated in larger cohorts.

\section{Acknowledgements}

The authors wish to thank all investigators of the PREP study in France, Belgium and Switzerland who contributed to the recruitment of the patients in the study.

\section{References}

1 Goldhaber SZ, Visani L, De Rosa M. Acute pulmonary embolism: clinical outcomes in the International Cooperative Pulmonary Embolism Registry (ICOPER). Lancet 1999; 353: 1386-1389.

2 Torbicki A, Perrier A, Konstantinides S, et al. Guidelines on the diagnosis and management of acute pulmonary embolism: the task force for the diagnosis and management of acute pulmonary embolism of the European Society of Cardiology (ESC). Eur Heart J 2008; 29: 2276-2315.

3 Aujesky D, Obrosky DS, Stone RA, et al. Derivation and validation of a prognostic model for pulmonary embolism. Am J Respir Crit Care Med 2005; 172: 1041-1046.

4 Aujesky D, Perrier A, Roy PM, et al. Validation of a clinical prognostic model to identify low-risk patients with pulmonary embolism. J Intern Med 2007; 261: 597-604.

5 Donze J, Le Gal G, Fine MJ, et al. Prospective validation of the pulmonary embolism severity index. A clinical prognostic model for pulmonary embolism. Thromb Haemost 2008; 100: 943-948. 
Jimenez D, Aujesky D, Moores L, et al. Simplification of the pulmonary embolism severity index for prognostication in patients with acute symptomatic pulmonary embolism. Arch Intern Med 2010; 170: $1383-1389$.

7 Becattini C, Vedovati MC, Agnelli G. Prognostic value of troponins in acute pulmonary embolism: a meta-analysis. Circulation 2007; 116: 427-433.

8 Sanchez O, Trinquart L, Caille V, et al. Prognostic factors for pulmonary embolism: the prep study, a prospective multicenter cohort study. Am J Respir Crit Care Med 2010; 181: 168-173.

9 Sanchez O, Trinquart L, Colombet I, et al. Prognostic value of right ventricular dysfunction in patients with haemodynamically stable pulmonary embolism: a systematic review. Eur Heart J 2008; 29: $1569-1577$.

10 Jimenez D, Lobo JL, Monreal M, et al. Prognostic significance of multidetector computed tomography in normotensive patients with pulmonary embolism: rationale, methodology and reproducibility for the PROTECT study. J Thromb Thrombolysis 2012; 34: 187-192.

11 Klok FA, Mos IC, Huisman MV. Brain-type natriuretic peptide levels in the prediction of adverse outcome in patients with pulmonary embolism: a systematic review and meta-analysis. Am J Respir Crit Care Med 2008; 178 : 425-430.

12 Lankeit M, Gómez V, Wagner C, et al. A strategy combining imaging and laboratory biomarkers in comparison with a simplified clinical score for risk stratification of patients with acute pulmonary embolism. Chest 2012; 141: 916-922.

13 Aujesky D, Roy PM, Verschuren F, et al. Outpatient versus inpatient treatment for patients with acute pulmonary embolism: an international, open-label, randomised, non-inferiority trial. Lancet 2011; 378: 41-48.

14 Moores L, Aujesky D, Jiménez D, et al. Pulmonary embolism severity index and troponin testing for the selection of low-risk patients with acute symptomatic pulmonary embolism. J Thromb Haemost 2010; 8: 517-522.

15 Lankeit M, Jimenez D, Kostrubiec M, et al. Predictive value of the high-sensitivity troponin T assay and the simplified pulmonary embolism severity index in hemodynamically stable patients with acute pulmonary embolism: a prospective validation study. Circulation 2011; 124: 2716-2724.

16 Vanni S, Nazerian P, Pepe G, et al. Comparison of two prognostic models for acute pulmonary embolism: clinical vs. right ventricular dysfunction-guided approach. J Thromb Haemost 2011; 9: 1916-1923. 